\title{
ACTIVATED TRANSPORT IN MAGNETIC-FIELD INDUCED INSULATING PHASE IN TWO-DIMENSIONAL ELECTRON GAS IN InGaAs/InP HETEROSTRUCTURES
}

\author{
B. PőDöR* , G. GomBos
}

Research Institute for Technical Physics of the Hungarian Academy of Sciences

Budapest, Hungary

G. REMÉNYI

CNRS Centre de Recherche sur les Tres Basses Températures et Laboratoire des Champs Magnétiques Intenses, Grenoble, France

$$
\text { Gy. Kovács }
$$

Applied Physics Research Group, Department of General Physics Loránd Eötvös University, Budapest, Hungary

\section{I.G. SAVEL'Ev aND S.V. Novikov}

Ioffe Physical-Technical Institute of the Russian Academy of Sciences St. Petersburg, Russia

We report on experiments on low temperature (millikelvin range) activated magnetotransport on low-density two-dimensional electron systems in InGaAs/InP for Landau level filling factors $0.25 \leq \nu \leq 0.55$. The activation energy increases approximately linearly with decreasing filling factor. The observations are discussed in the light of the formation of the Wigner solid.

PACS numbers: $73.20 . \mathrm{Dx}$

\section{Introduction}

In recent years the occurrence of insulating phases in two-dimensional electron systems at semiconductor interfaces in high magnetic field has been the subject of intensive research in connection with the still elusive Wigner crystallization. At low temperature and small Landau level filling factor $\left(\nu=n_{\mathrm{s}} h / e B\right)$, the ground state of a two-dimensional electron system (2DES) with small disorder is expected

*E-mail: podor@mufi.hu. 
to be an electron solid (Wigner crystal). The Wigner transition was observed in nearly perfect GaAlAs/GaAs heterostructures [1-5], and quite recently in the InGaAs/InP heterostructure too [6, 7]. In GaAlAs/GaAs the value of the critical filling factor $\nu_{\mathrm{c}}$ at $T=0 \mathrm{~K}$, below which Wigner crystallization occurs, was about $0.2[1,2,4]$ and 0.28 [3], while in $\operatorname{InGaAs} / \operatorname{InP}$ a higher value of about $\nu_{c}=0.49$ was obtained [7]. Experiments performed on the GaAlAs/GaAs system indicated that the solid exhibits activated [8-10] and non-linear transport properties $[1,2,4,5]$, attributed to the pinning on the random potential introduced by defects and impurities. Similar non-linear transport properties were also observed in the InGaAs/InP system by the present authors [6,7].

Here we report on the observation of activated magnetotransport in low-density, low-mobility two-dimensional electron gas (2DEG) in lattice matched InGaAs/InP single heterostructures in the range of Landau level filling factors of $0.25 \leq \nu \leq 0.55$. Experimental results concerning the activation energy $E_{\mathrm{A}}$ in the longitudinal resistance $\rho_{x x}$ and its dependence on the filling factor are presented and discussed.

\section{Experimental}

The samples were liquid phase epitaxial $\operatorname{In}_{0.53} \mathrm{Ga}_{0.47} \mathrm{As} / \mathrm{InP}$ heterostructures $[6,7]$. The electron density and mobility were $(0.3-1.5) \times 10^{11} \mathrm{~cm}^{-2}$ and (1-5) $\times 10^{4} \mathrm{~cm}^{2} /(\mathrm{V} \mathrm{s})$ respectively. Sample parameters are collected in Table. Persistent photoconductivity was used to control the 2DEG density. Most of the

TABLE

Parameters of the 2DEG in the In GaAs/InP samples.

\begin{tabular}{l|c|c|c|c|c|c}
\hline \hline Sample & $\begin{array}{c}n_{\mathbf{s}} \\
{\left[\mathrm{cm}^{-2}\right]}\end{array}$ & $\begin{array}{c}\mu \\
{\left[\mathrm{cm}^{2} /(\mathrm{V} \mathrm{s})\right]}\end{array}$ & $\begin{array}{c}B(\nu=1) \\
{[\mathrm{T}]}\end{array}$ & $\begin{array}{c}E_{\mathrm{a} 0} \\
{[\mathrm{~K}]}\end{array}$ & $\begin{array}{c}\alpha \\
{[\mathrm{K}]}\end{array}$ & $\nu_{\mathrm{c}}$ \\
\hline 1 & $3.3 \times 10^{10}$ & $1.6 \times 10^{4}$ & 1.36 & 24.4 & 34.9 & 0.70 \\
2 & $1.2 \times 10^{11}$ & $2.9 \times 10^{4}$ & 4.96 & 17.8 & 37.9 & 0.47 \\
$3^{*}$ & $7.5 \times 10^{10}$ & $5.2 \times 10^{4}$ & 3.10 & 15.0 & 34.8 & 0.43 \\
\hline
\end{tabular}

*Sample 1 after illumination (persistent photoconductivity mode).

magnetotransport measurements were carried out in a $\mathrm{He}^{3}-\mathrm{He}^{4}$ dilution refrigerator fitted into the bore of a resistive magnet capable of reaching $22 \mathrm{~T}$. The temperature range was 40 to $800 \mathrm{mK}$. Both conventional dc and $(8 \mathrm{~Hz})$ lock-in measuring techniques were used, and both two- and four-terminal resistivity measurements were performed.

\section{Results and discussion}

Figure 1 shows typical longitudinal resistivity versus reciprocal temperature plots for several magnetic fields corresponding to filling factors $\nu \leq 0.5$, where the resistivity began to increase dramatically with increasing magnetic field, reaching values in excess of about hundred times $h / e^{2}$. As can be seen in the figure, the 


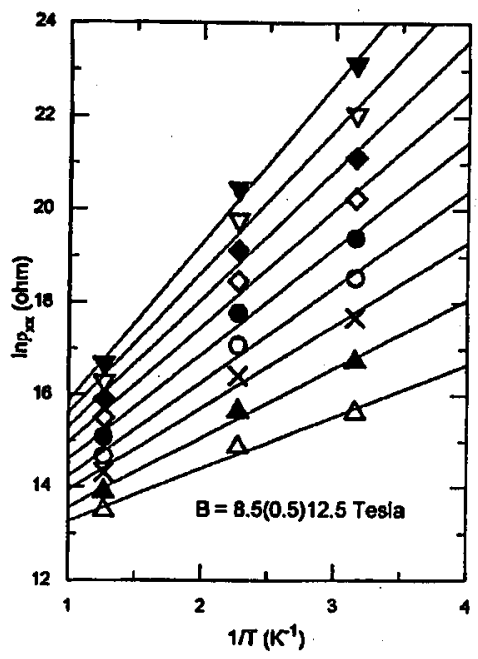

Fig. 1. Longitudinal resistance $\rho_{x x}$ versus the reciprocal temperature for sample 3 with $n_{s}=3.5 \times 10^{10} \mathrm{~cm}^{-2}$. The magnetic fields correspond to the range of filling factors from 0.25 to 0.37 .

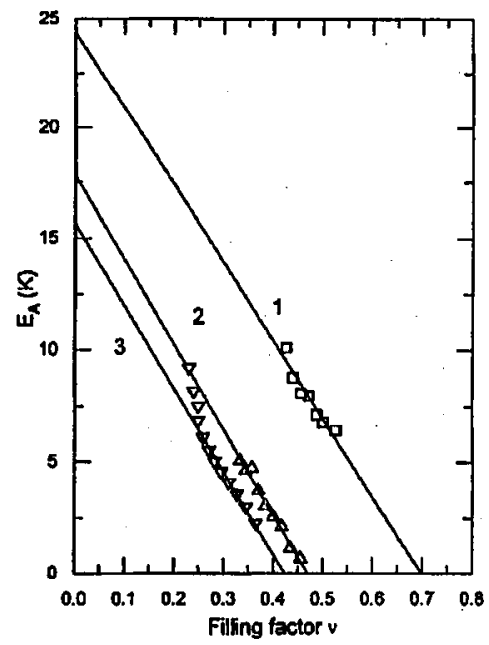

Fig. 2. Dependence of the activation energy $E_{\mathrm{A}}$ on the filling factor $\nu$. 2DEG densities for samples 1 through 3 are $1.2 \times 10^{11}, 7.5 \times 10^{10}$, and $3.3 \times 10^{10} \mathrm{~cm}^{-2}$ respectively. Linear fits indicated correspond to $E_{\mathrm{A}}=E_{\mathrm{a} 0}-\alpha \nu$.

resistivity displays an activated behaviour for temperatures above about $200 \mathrm{mK}$. The activation energies were determined according to $\rho_{x x}=\rho_{0} \exp \left(E_{\mathrm{A}} / 2 k T\right)$. In Fig. 2 the activation energies determined for three samples are plotted as a function of the filling factor $\nu . E_{\mathrm{A}}$ decreases roughly linearly with the filling factor $\nu$ with similar slopes for all the three samples. Parameters obtained from a fit to the equation $E_{\mathrm{A}}=E_{\mathrm{a} 0}-\alpha \nu$ are listed in Table. The intercept with the horizontal axis 
$\left(\nu_{\mathrm{c}}\right)$ marks the onset of the temperature dependence of $\rho_{x x}$. It can be observed that the higher is the disorder (as quantified by the value of the 2DEG mobility), the larger are the extrapolated activation energy $E_{\mathrm{a} 0}=E_{\mathrm{A}}(\nu=1)$ and the critical filling factor $\nu_{\mathrm{c}}$ for the onset of the activated transport.

The data for the onset obtained here for various samples at various magnetic fields can be extrapolated to infinite magnetic field values (assumed in all theoretical models) using $\nu=n_{\mathrm{s}} / e B$ as suggested in [8]. As is shown in Fig. 3, a limiting value of $\nu=0.37$ is obtained for $B \rightarrow \infty$. This value is about twice of the corresponding value $(\nu=0.19)$ obtained for high mobility (low-disorder) GaAlAs/GaAs [8], which in turn is identified there with the occurrence of the Wigner solidification.

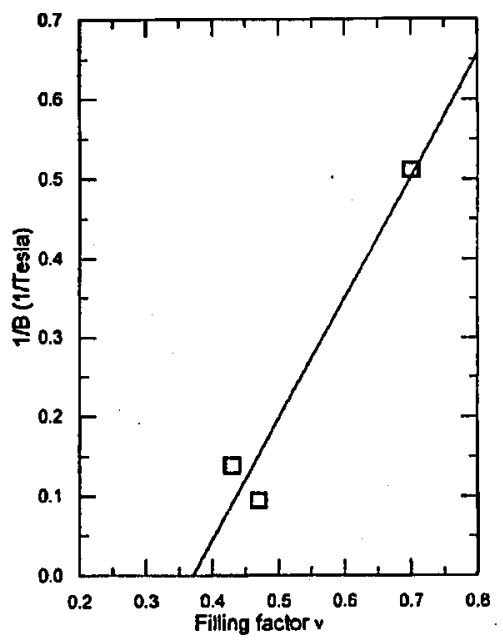

Fig. 3. Extrapolation of the onset $\nu\left(E_{\mathrm{A}}\right)$ to infinite $B$ using $\nu=n_{\mathrm{s}} h / e B$.

In the millikelvin range all samples exhibited non-linear current voltage characteristics with a filling factor dependent plateau and threshold voltage. Two terminal resistance increased dramatically below about $\nu=0.5$, and a noisy resistance roll-off was also observed in the ac measurements. The temperature dependence of the $I-V$ characteristics revealed saturation for higher temperatures with a lower threshold voltage. All these phenomena can be considered as signatures of the Wigner solidification $[6,7]$.

Theoretically expected ground state of the 2DES at low temperatures depends on the strength of the disorder. The stronger is the disorder the more glassy is the Wigner solid pinned by the disorder. In low mobility samples strongly pinned Wigner solid is expected with the onset of activated transport at higher values of $\nu$ than in the high mobility case. Our experimental results are in a perfect qualitative agreement with these predictions. In the lowest concentration samples we found $\nu=0.7$, much greater value than reported for high mobility GaAlAs/GaAs heterostructures. 


\section{Conclusions and acknowledgments}

In summary, we observed activated transport in the small filling factor regime in 2DEG in InGaAs/InP. New results for the activation energy were obtained. The activation energy increased with decreasing filling factors. The critical filling factor for the onset of the activated transport is tentatively identified with the Wigner solidification of the 2DEG.

The high magnetic field measurements were performed in the CNRS/ Max-Planck-Institut High Magnetic Field Laboratory, Grenoble, under the project SE1295. Gy.K., I.G.S., and B.P. express their gratitude for the hospitality of the CNRS. Partial support was also made available from the Hungarian National Research Fund projects No. OTKA/14094 and OTKA/22894.

\section{References}

[1] E.Y. Andrei, G. Deville, D.C. Glattli, F.I.B. Williams, E. Paris, B. Etienne, Phys. Rev. Lett. 60, 2765 (1988).

[2] V.J. Goldman, M. Santos, M. Shayegan, J.E. Cunningham, Phys. Rev. Lett. 65, 2189 (1990).

[3] H. Buhmann, W. Joss, K. von Klitzing, I.V. Kukushkin, A.S. Plaut, G. Martinez, K. Ploog, V.B. Timofeev, Phys. Rev. Lett. 66, 926 (1991).

[4] F.I.B. Williams, G. Deville, D.C. Glattli, P.A. Wright, E.Y. Andrei, R.G. Clark, B. Etienne, C.T. Foxon, J.J. Harris, Surf. Sci. 263, 23 (1992).

[5] E.Y. Andrei, F.I.B. Williams, D.C. Glattli, G. Deville, in: The Physics of Low-Dimensional Semiconductor Structures, Ed. P. Butcher, Plenum Press, New York 1993, p. 499.

[6] Gy. Kovács, G. Reményi, G. Gombos, I.G. Savel'ev, A.M. Kreshchuk, N. Hegman, B. Pödör, Acta Phys. Pol. A 88, 783 (1995).

[7] G. Reményi, Gy. Kovács, I.G. Savel'ev, G. Gombos, B. Pödör, Czech. J. Phys. 46 (Suppl. 5), 2527 (1996).

[8] R.L. Willett, H.L. Stormer, D.C. Tsui, L.N. Pfeiffer, K.W. West, K.W. Baldwin, Phys. Rev. B 38, 7881 (1988).

[9] R.L. Willett, H.L. Stormer, D.C. Tsui, L.N. Pfeiffer, K.W. West, in: High Magnetic Fields in Semiconductor Physics II, Ed. G. Landwehr, Springer Series in Solid State Physics, Vol. 87, Springer, Berlin 1989, p. 153.

[10] A. Fukano, S. Kawaji, J. Wakabayashi, K. Hirakawa, H. Sakaki, T. Goto, Y. Koike, T. Fukase, in: High Magnetic Fields in the Physics of Semiconductors, Ed.

D. Heiman, World Scientific, Singapore 1996, p. 440. 